\title{
Regulation of arterial pressure by the paraventricular nucleus in conscious rats: interactions among glutamate, GABA, and nitric oxide
}

\author{
Marli C. Martins-Pinge ${ }^{1+}$, Patrick J. Mueller ${ }^{2,3+}$, C. Michael Foley ${ }^{2,3+}$, Cheryl M. Heesch ${ }^{2,3}$ and \\ Eileen M. Hasser ${ }^{2,3,4 *}$
}

${ }^{1}$ Department of Physiological Sciences, Center of Biological Sciences, State University of Londrina, Londrina, Brazil

2 Department of Biomedical Sciences, University of Missouri, Columbia, MO, USA

${ }^{3}$ Dalton Cardiovascular Research Center, University of Missouri, Columbia, MO, USA

${ }^{4}$ Department of Medical Pharmacology and Physiology, University of Missouri, Columbia, MO, USA

Edited by:

John W. Osborn, University

of Minnesota, USA

Reviewed by:

Roger Dampney, The University

of Sydney, Australia

Matthew Zahner, Pfizer Global

$R \& D, U S A$

${ }^{*}$ Correspondence:

Eileen M. Hasser, Dalton

Cardiovascular Research Center,

134 Research Park Drive,

University of Missouri, Columbia,

MO 65211-3300, USA.

e-mail: hassere@missouri.edu

${ }^{\dagger}$ Present address:

Marli C. Martins-Pinge, Department of Physiological Sciences, Center of Biological Sciences, State University of Londrina, Londrina, PR, Brazil.

Campus Universitario, s/n, Rodovia Celso Garcia Cid, Km 380, Portal de Versalles II, 86051-990, Londrina, PR, Brazil.

Patrick J. Mueller, Department of Physiology, Wayne State University School of Medicine, 540 E. Canfield, Detroit, MI 48201, USA.

C. Michael Foley, Cardiac Safety Scientist, Safety Assessment, Covance Laboratories, Inc., 3301 Kinsman Blvd., Madison, WI 53704, USA.
The paraventricular nucleus (PVN) of the hypothalamus is an important site for autonomic and neuroendocrine regulation. Experiments in anesthetized animals and in vitro indicate an interaction among gamma-aminobutyric acid (GABA), nitric oxide (NO), and glutamate in the PVN. The cardiovascular role of the PVN and interactions of these neurotransmitters in conscious animals have not been evaluated fully. In chronically instrumented conscious rats, mean arterial pressure (MAP) and heart rate $(\mathrm{HR})$ responses to microinjections $(100 \mathrm{nl})$ in the region of the PVN were tested. Bilateral blockade of ionotropic excitatory amino acid (EAA) receptors (kynurenic acid, Kyn) in the PVN produced small but significant decreases in MAP and HR. GABA receptor blockade (bicuculline, Bic), and inhibition of NO synthase [(NOS), N-(G)-monomethyl-L-arginine, L-NMMA] each increased MAP and HR. The NO donor sodium nitroprusside (SNP) produced depressor responses that were attenuated by Bic. NOS inhibition potentiated both pressor responses to the selective EAA agonist, N-methyl-D-aspartic acid (NMDA), and depressor responses to Kyn. Increases in MAP and HR due to Bic were blunted by prior blockade of EAA receptors. Thus, pressor responses to GABA blockade require EAA receptors and GABA neurotransmission contributes to NO inhibition. Tonic excitatory effects of glutamate in the PVN are tonically attenuated by NO. These data demonstrate that, in the PVN of conscious rats, GABA, glutamate, and NO interact in a complex fashion to regulate arterial pressure and HR under normal conditions.

Keywords: autonomic, sympathetic, NMDA, PVN, hypothalamus

\section{INTRODUCTION}

The paraventricular nucleus (PVN) of the hypothalamus is a critical site of integration for autonomic- and neuroendocrinemediated cardiovascular responses (Swanson and Sawchenko, 1983). In addition to its role in the hypothalamoneurohypophysial system, the PVN is involved in regulation of the autonomic nervous system (Pyner and Coote, 2000; Patel et al., 2001; Li and Patel, 2003; Coote, 2004). Morphological and electrophysiological studies have shown that the PVN is reciprocally connected to regions of the brain that are involved in cardiovascular regulation (Saper et al., 1976; Swanson and Sawchenko, 1983). Importantly, the PVN projects to both the intermediolateral cell column of the spinal cord and the rostral ventrolateral medulla
(RVLM), regions critical in control of the sympathetic nervous system (Badoer, 1996; Pyner and Coote, 2000; Hardy, 2001).

Nitric oxide (NO) has been shown to act as a gaseous neuromodulator to influence synaptic function in the central nervous system (Bredt et al., 1990; Hirooka et al., 1996; Martins-Pinge et al., 1997; Zanzinger, 1999). NO synthase (NOS) is expressed in the PVN (Zhang et al., 1998; Kantzides and Badoer, 2005; Mueller et al., 2006) and NO appears to play a role in endocrine and autonomic regulation of cardiovascular responses. In vitro studies indicate that neuronal activity within the PVN is modulated by NO (Li et al., 2003; Stern, 2004). In addition, administration of an NO donor into the PVN decreases renal sympathetic nerve discharge, arterial pressure, and heart rate (HR; Horn et al., 
1994; Zhang and Patel, 1998). Conversely, microinjection of NOS blockers into the PVN produces pressor and sympathoexcitatory responses (Zhang and Patel, 1998; Wang et al., 2005). These data, primarily from anesthetized animals, suggest that NO has a tonic effect within the PVN to inhibit resting sympathetic activity and arterial pressure.

The effects of NO within the PVN appear to involve a complex interaction with the neurotransmitters glutamate and gammaaminobutyric acid (GABA). It has been proposed that activation of the NMDA subtype of ionotropic glutamate receptors increases release of $\mathrm{NO}$ in the PVN, which then negatively modulates NMDA-mediated increases in sympathetic nerve discharge (Li et al., 2001). Depressor and sympathoinhibitory responses to NO donors are blocked by the $\mathrm{GABA}_{\mathrm{A}}$ receptor antagonist bicuculline (Bic; Zhang and Patel, 1998). This is consistent with the concept that NO may blunt responses to exogenous NMDA by increasing GABA transmission. However, it is not known whether endogenous NO tonically modulates basal excitatory effects of glutamate within the PVN.

The role of NO is known to differ dramatically during different levels of neuronal activity and under a variety of physiological and pathophysiological conditions (Villar et al., 1994; DiCarlo et al., 2002; Felder et al., 2003; Li and Patel, 2003; Mueller et al., 2003, 2006; Heesch et al., 2009). However, the interactions among NO, GABA, and glutamate have been studied primarily in anesthetized animals (Zhang and Patel, 1998; Li et al., 2001; Patel et al., 2001; Akine et al., 2003). Anesthesia is well-known to alter neurotransmission, including both GABAergic and glutamatergic neurotransmission (Franks and Lieb, 1982; Jin et al., 2009; Olsen and Li, 2011), and autonomic and cardiovascular regulation (Schadt and Ludbrook, 1991; Moffitt et al., 1998, 1999; Araújo et al., 1999; Sakima et al., 2000; Machado, 2001). Furthermore, anesthesia alters cardiovascular responses mediated by the PVN (Kannan et al., 1987, 1989). Given this evidence, it is apparent that GABA, glutamate, and NO function may differ in the conscious state. Therefore, it is critical to evaluate the relative roles of GABA, glutamate, and NO, and their interactions, in conscious animals in order to determine the importance of these transmitters in the PVN in control of the cardiovascular system in the conscious state.

This study in conscious rats tested the hypothesis that, in regard to control of arterial pressure, both NO and GABA in the PVN are tonically inhibitory, while glutamate is tonically excitatory. We also hypothesized that the tonic inhibitory effects of $\mathrm{NO}$ require GABAergic mechanisms within the PVN. Finally, we tested the hypothesis that endogenous NO modulates the tonic excitatory effects of glutamate.

\section{EXPERIMENTAL PROCEDURES}

All procedures were performed according to the guidelines stated in the National Institutes of Health "Guide for the Care and Use of Laboratory Animals." All protocols were approved by the University of Missouri-Columbia Animal Care and Use Committee. Twenty-nine male Sprague-Dawley rats (280-350 g, Harlan Sprague Dawley, Indianapolis, IN) were used in these studies. The rats were allowed to adapt in house for at least 1 week before any instrumentation. Rats were allowed access to water and food (Formulab Diet, $0.28 \%$ sodium, Purina, St. Louis, MO) ad libitum.

\section{INSTRUMENTATION}

Initially, rats were anesthetized with Isoflurane [AErane, Baxter, Deerfield, IL (5\% in $100 \% \mathrm{O}_{2}, 2 \mathrm{~L}$ per minute for induction and maintenance at 2-2.5\%)], placed in a stereotaxic apparatus (Kopf, Tujunga, CA) and using aseptic technique bilateral guide cannulae (22 gauge) directed to the region of the PVN were implanted. Coordinates for cannulae placement were similar to previous work (Kenney et al., 2001; Li et al., 2001; Zhang et al., 2002; Chen and Toney, 2003): $1.8 \mathrm{~mm}$ posterior to bregma, $0.5 \mathrm{~mm}$ lateral to midline, and $7.6 \mathrm{~mm}$ below the surface, with the incisor bar set to $5 \mathrm{~mm}$ above the interaural plane and the cannula vertical. Studies from our laboratories have shown similar coordinates to produce injections sites localized to the PVN (Kvochina et al., 2009; King et al., 2012). Rats were allowed to recover for 12 days, anesthetized again with Isoflurane and aseptically instrumented with arterial and venous catheters for measurement of arterial pressure and $\mathrm{HR}$ and injection of drugs, respectively. The catheters were routed subcutaneously to the nape of the neck, exteriorized, and secured to the skin with suture; catheters were filled with heparinized saline $(100 \mathrm{U} / \mathrm{ml})$ and sealed with a stylet. Rats were allowed to recover for 2 days before the beginning of experiments.

\section{EXPERIMENTAL PROTOCOLS}

On the day of experimentation, arterial pressure, mean arterial pressure (MAP), and HR were monitored (PowerLab; ADInstruments, Colorado Springs, CO) for at least $30 \mathrm{~min}$ to ensure stable baseline parameters prior to any experimental manipulation. Microinjections were made using stainless steel injectors ( 33 gauge) connected by $\mathrm{PE}$ tubing to a $0.5 \mu \mathrm{l}$ syringe (Hamilton, Reno, NV). Injectors were filled with the appropriate solution and inserted into the guide cannulae prior to injection. Protocols designed to evaluate tonic effects of endogenous transmitters utilized bilateral microinjections of appropriate agents to block the effects of the specific transmitter. Because the time course of the agonists used is relatively short, protocols examining effects of agonists utilized unilateral microinjections. As in other studies (Chen and Toney, 2003; Chen et al., 2003; Li and Pan, 2007a), the PVN initially was identified in all rats by pressor responses to $\mathrm{Bic}(1 \mathrm{mM})$ with mild or no behavioral response.

\section{Tonic excitation of the PVN}

Tonic effects on MAP and HR of ionotropic glutamate receptor activation in the PVN in conscious rats were evaluated by examining responses to bilateral microinjection of kynurenic acid [Kyn, 40 mM; 100 nl, (Li and Pan, 2007a)], a general ionotropic glutamate receptor antagonist. In control experiments, similar volumes of saline $(0.9 \%)$ were injected bilaterally.

\section{Tonic inhibition of the PVN}

These protocols examined the tonic effects of $\mathrm{NO}$ and $\mathrm{GABA}_{\mathrm{A}}$ receptor activation within the $\mathrm{PVN}$ on cardiovascular function. Bilateral microinjections $(100 \mathrm{nl})$ of L-NMMA [N-(G)monomethyl-L-arginine, $2 \mathrm{mM}$ (Li et al., 2001), a NOS inhibitor, or Bic $1 \mathrm{mM}$, (Li et al., 2006)], a GABA $\mathrm{A}$ receptor antagonist, were made in the PVN and hemodynamic responses monitored. 


\section{Transmitter interactions}

To examine the interactions among NO, GABA, and glutamate in the PVN of conscious rats, the response to manipulation of the different receptor systems was evaluated in the presence and absence of other systems. Hemodynamic responses to unilateral microinjection of the NO donor sodium nitroprusside [SNP, $1 \mathrm{M} ; 100 \mathrm{nl}$, (Zhang and Patel, 1998) were evaluated alone and in the presence of Bic $(1 \mathrm{mM} ; 100 \mathrm{nl})$, or saline $(0.9 \% ; 100 \mathrm{nl})]$. Similarly, the effects of unilateral microinjections of the selective ionotropic glutamate receptor agonist N-methyl-D-aspartate [NMDA, $1 \mathrm{mM}$; $100 \mathrm{nl}$, (Li et al., 2001)] were evaluated alone and in the presence of the NOS inhibitor L-NMMA ( $2 \mathrm{mM} ; 100 \mathrm{nl})$.

To examine the interaction of tonic endogenous ionotropic glutamate receptor activation with endogenous NO, the response to bilateral PVN injection of Kyn $(40 \mathrm{mM}, 100 \mathrm{nl})$ was evaluated in the presence and absence of inhibition of NOS with L-NMMA $(2 \mathrm{mM} ; 100 \mathrm{nl})$. Finally, the interaction of endogenous glutamate and GABA was evaluated. Hemodynamic effects of bilateral blockade of $\mathrm{GABA}_{\mathrm{A}}$ receptors by microinjection of Bic $(1 \mathrm{mM}$; $100 \mathrm{nl}$ ) were examined alone and in the presence of blockade of ionotropic glutamate receptors with Kyn (40 mM; $100 \mathrm{nl})$.

The time allowed for recovery following the initial administration of a specific agent was based on previous studies showing recovery, as well as repeatable responses (Li et al., 2001; Chen and Toney, 2003; Chen et al., 2003). The second administration of each drug was made $5 \mathrm{~min}$ after antagonist administration. A maximum of 3 drug administrations was performed in each PVN and drugs were applied using a microsyringe connected by PE tubing to an injector. At the end of the experiment, Pontamine sky blue dye $(2 \% ; 100 \mathrm{nl})$ was microinjected into the experimental site of the brain for subsequent histological estimation of injection sites.

At the end of the experiment, rats were euthanized by overdose of sodium pentobarbital; guide cannulae were detached from their cemented position on the skull and brains were fixed in $4 \%$ formaldehyde for at least $48 \mathrm{~h}$. Serial coronal sections $(40 \mu \mathrm{m})$ were cut using a cryostat, thaw mounted on microscope slides and stained with 1\% aqueous neutral red. Figure 1 shows the injection site within the PVN in one rat. Unfortunately, in most rats removing the guide cannulae prior to fixation of the brains produced non-specific damage. However, injection tracts, the rostral-caudal spread of the dye and dye spots were confined to the brain parenchyma in regions consistent with PVN injection sites reported in previous studies from our laboratories and others (Martin and Haywood, 1998; Kenney et al., 2001; Li et al., 2001; Chen et al., 2003; Kvochina et al., 2009; King et al., 2012).

\section{DRUGS}

Bic methiodide, SNP, L-NMMA, and Kyn were obtained from Sigma Chemical (St. Louis, MO). NMDA was obtained from Tocris (St. Louis, MO). With the exception of Kyn, all drugs were dissolved in sterile saline. Kyn was first dissolved in a few drops of $1 \mathrm{~N} \mathrm{NaOH}$ before being diluted in saline. All drugs were $\mathrm{pH}$ adjusted to 7.3-7.5 and filtered before microinjection. Doses of drugs for microinjection were taken from previous studies (Martin et al., 1991; Zhang and Patel, 1998; Chen and Toney, 2001; Chen et al., 2003; Li et al., 2006; Li and Pan, 2007a,b). Most

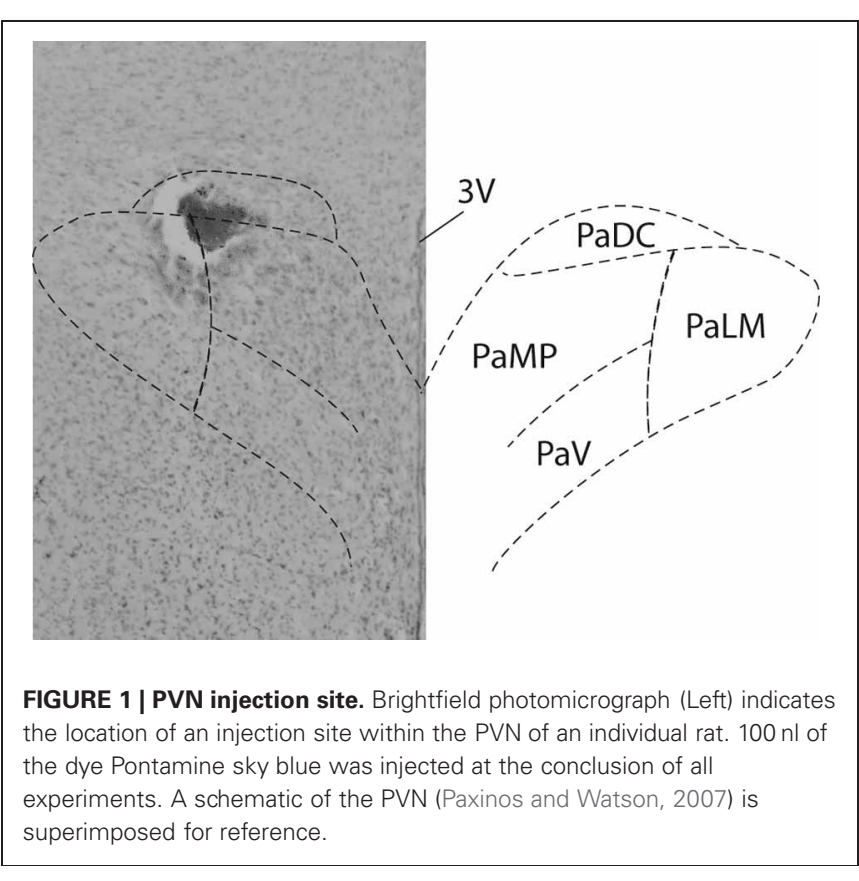

drugs used for microinjection were relatively short-acting with recovery typically within $30-45 \mathrm{~min}$ or less.

\section{ANALYSIS OF DATA}

All results are expressed as mean \pm SEM. Responses to drugs are expressed as the difference between the basal value and the peak increase or decrease following each dose of drug. Student's twotailed paired $t$-test was used for comparison of hemodynamic parameters between baseline conditions and after drug. We also used Student's two-tailed paired $t$-test to compare the response (change) to two different drugs or to a given drug in the presence and absence of another perturbation in the same rat. $P<0.05$ was considered to indicate statistical significance.

\section{RESULTS}

\section{BASELINE HEMODYNAMIC PARAMETERS}

There were no significant differences in baseline hemodynamic parameters before any protocols involving microinjection of substances into the PVN in conscious rats (Table 1). Average baseline $\mathrm{MAP}$ was $117 \pm 2 \mathrm{mmHg}$, and $\mathrm{HR}$ was $347 \pm 4 \mathrm{bpm}(n=29)$. As described by others (Martin et al., 1991), after microinjection of Bic and NMDA into the PVN, we often observed mild behavioral responses, including alerting and facial grooming. These behaviors were not aggressive and were distinctly different from the defense responses described after activation of the posterior hypothalamus (Shekhar and DiMicco, 1987; Wible et al., 1988).

\section{TONIC IONOTROPIC GLUTAMATERGIC EXCITATION WITHIN THE PVN}

Figure $2 \mathbf{A}$ is an original record showing MAP and HR responses to bilateral microinjection of the broad spectrum excitatory amino acid (EAA) receptor antagonist, Kyn, in the PVN of a conscious rat. Bilateral blockade of ionotropic glutamate receptors with Kyn $(n=4)$ produced a small but significant decrease in MAP $(-6 \pm 2 \mathrm{mmHg})$ and $\mathrm{HR}(-15 \pm 3 \mathrm{bpm})$; Table 1. 
Table 1 | Mean arterial pressure (MAP) and heart rate (HR) before and after drugs microinjected in the PVN.

\begin{tabular}{llll}
\hline Groups & \multicolumn{2}{c}{ Before } & \multicolumn{2}{c}{ After } \\
\cline { 2 - 4 } & MAP (mmHg) & HR (bpm) & MAP (mmHg) \\
\hline Bic $(n=7)$ & $119 \pm 6$ & $346 \pm 9$ & $141 \pm 5^{*}$ \\
L-NMMA $(n=6)$ & $113 \pm 5$ & $332 \pm 10$ & $133 \pm 2^{*}$ \\
Kyn $(n=4)$ & $128 \pm 6$ & $371 \pm 7$ & $121 \pm 4^{*}$ \\
SNP ( $n=6)$ & $120 \pm 2$ & $344 \pm 16$ & $107 \pm 3^{*}$ \\
SNP (after Bic; $n=6)$ & $121 \pm 4$ & $355 \pm 17$ & $114 \pm 5^{*}$ \\
Bic (after Kyn; $n=6)$ & $125 \pm 4$ & $357 \pm 14$ & $134 \pm 5^{*}$ \\
Kyn (after L-NMMA; $n=4)$ & $134 \pm 8$ & $383 \pm 8$ & $121 \pm 7^{*}$ \\
NMDA (n=4) & $124 \pm 2$ & $361 \pm 12$ & $135 \pm 2^{*}$ \\
NMDA (after L-NMMA; $n=4)$ & $124 \pm 3$ & $364 \pm 5.1$ & $142 \pm 2^{*}$ \\
\hline
\end{tabular}

${ }^{*} p<0.05$ compared to values before microinjection.

Bilateral microinjection of physiological saline $(n=5)$ produced no significant effect on MAP $(-1 \pm 1 \mathrm{mmHg})$ or $\mathrm{HR}$ $(-4 \pm 17 \mathrm{bpm})$.

\section{TONIC INHIBITION WITHIN THE PVN}

Original tracings of hemodynamic responses to bilateral microinjection of the $\mathrm{GABA}_{\mathrm{A}}$ receptor antagonist, Bic, or the NOS inhibitor, L-NMMA, into the PVN of individual conscious rats are shown in Figures 2B,C, respectively. Group data (Figure 3, Table 1) indicate that bilateral disinhibition of the PVN by microinjection of Bic $(n=7)$ produced a significant increase in both MAP and HR. Bilateral blockade of NOS in the PVN with L-NMMA $(n=6)$ also induced a pressor response accompanied by an increase in HR. The pressor responses to Bic and L-NMMA were not significantly different, but the HR effects were significantly greater for Bic.

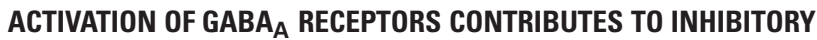 EFFECTS OF NITRIC OXIDE IN THE PVN OF CONSCIOUS RATS}

Initially, responses to two concentrations of the NO donor, SNP, were determined and repeatability of the response to multiple injections of SNP was tested. Unilateral microinjection of SNP $(0.5 \mathrm{M}$ and $1 \mathrm{M} ; n=5)$ into the PVN produced rapid dose-related decreases in MAP $(-11 \pm 2 \mathrm{mmHg}$ and $-17 \pm 3 \mathrm{mmHg}$, respectively). The depressor response was associated with tachycardia (51 \pm 15 and $54 \pm 3 \mathrm{bpm}$, respectively). Responses to unilateral microinjection of SNP $(0.5 \mathrm{M})$ were repeatable and not altered after microinjection of saline into the PVN (MAP: $-8 \pm 2$ vs. $-10 \pm 2$ mmHg; HR: $45 \pm 5$ vs. $32 \pm 8)$.

In separate rats $(n=6)$ responses to SNP $(1 \mathrm{M})$ were determined before and after injection of Bic (Figure 4, Table 1). Unilateral blockade of $\mathrm{GABA}_{\mathrm{A}}$ receptors with Bic initially tended to produce small increases in MAP and HR; however, 5 min later hemodynamic parameters had returned to control. Importantly, baseline MAP and HR before injection of SNP alone or injection after unilateral Bic were not statistically different (Table 1). In the presence of $\mathrm{Bic}$, the MAP response to SNP was attenuated by $\sim 50 \%$ (Figure 4 ). The tachycardic response produced by SNP was not altered by previous Bic microinjection.

\section{NITRIC OXIDE ATTENUATES TONIC GLUTAMATERGIC EXCITATION OF THE PVN}

As in the previous experiments, bilateral microinjection of Kyn into the PVN produced small but significant decreases in MAP and HR (Figure 5). After a $45 \mathrm{~min}$ recovery period, bilateral microinjection of Kyn was repeated in the presence of bilateral NOS blockade (L-NMMA). The baseline MAP and HR 5 min after L-NMMA, immediately before the second Kyn microinjection, were not different from the baseline before Kyn alone (Table 1). L-NMMA enhanced the depressor effect of ionotropic glutamate receptor blockade on MAP without significantly altering the HR responses (Figure 5).

Unilateral microinjection of the ionotropic glutamate agonist, NMDA, into the PVN of conscious rats produced an increase in MAP and HR (Figure 6, Table 1). Unilateral microinjection of L-NMMA did not alter baseline cardiovascular parameters. However, during NOS inhibition the pressor response to NMDA was significantly augmented (Figure 6), consistent with a role of NO to blunt the excitatory effects of NMDA receptor activation. The tachycardia in response to NMDA was not significantly altered by L-NMMA.

\section{TONIC GLUTAMATERGIC INPUT CONTRIBUTES TO EXCITATION OF THE

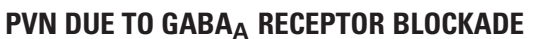

Figure 7 contains mean data demonstrating responses to bilateral Bic before and after ionotropic glutamate receptor blockade. Bilateral PVN microinjection of Bic produced significant increases in MAP and HR. After recovery, Kyn was injected bilaterally, and $5 \mathrm{~min}$ later Bic was injected a second time. Following ionotropic glutamate receptor blockade, the pressor and tachycardic responses to Bic were significantly attenuated.

\section{DISCUSSION}

PURPOSE

This study was designed to examine, in conscious rats, the influence of the PVN on arterial pressure and HR and to evaluate potential interactions of NO, glutamate, and GABA in mediating these effects. We also tested the hypothesis that endogenously released NO blunts the tonic excitatory effects of glutamate within the PVN. The use of conscious rats is an important aspect of 


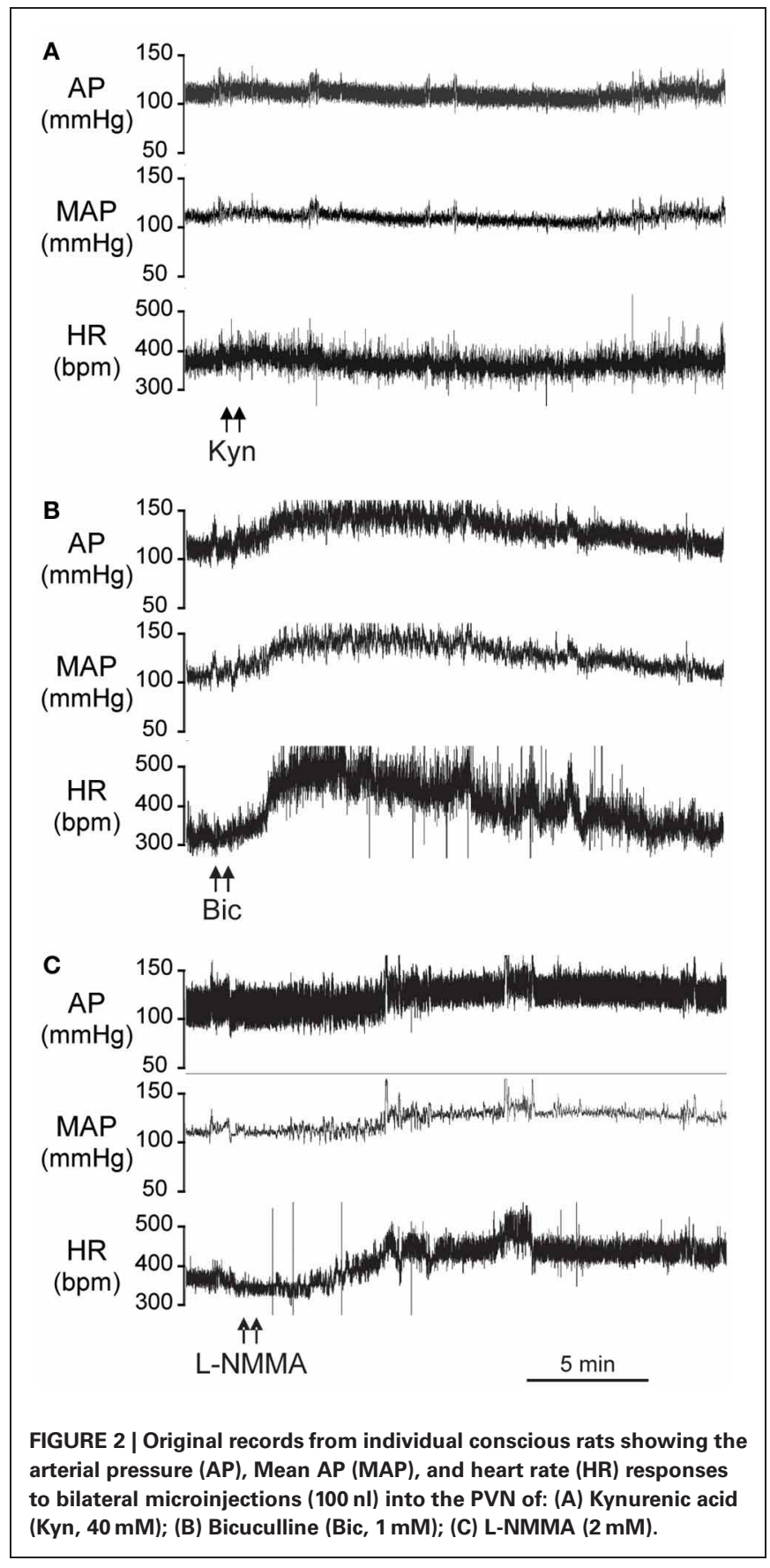

these studies in that anesthesia is well-known to alter neurotransmission, autonomic and cardiovascular function. Blockade of ionotropic glutamate receptors with Kyn in the region of the PVN produced a small but significant decrease in MAP and HR, suggesting that, in conscious rats, ionotropic glutamatergic transmission in the PVN participates in tonic excitatory drive to the vasculature and heart. Furthermore, the increase in MAP and $\mathrm{HR}$ due to bilateral blockade of either $\mathrm{GABA}_{\mathrm{A}}$ receptors or inhibition of NOS indicates that in the conscious state, similar to anesthetized animals, the PVN is under tonic inhibition by both GABA via $\mathrm{GABA}_{\mathrm{A}}$ receptors and by NO. The inhibitory effects
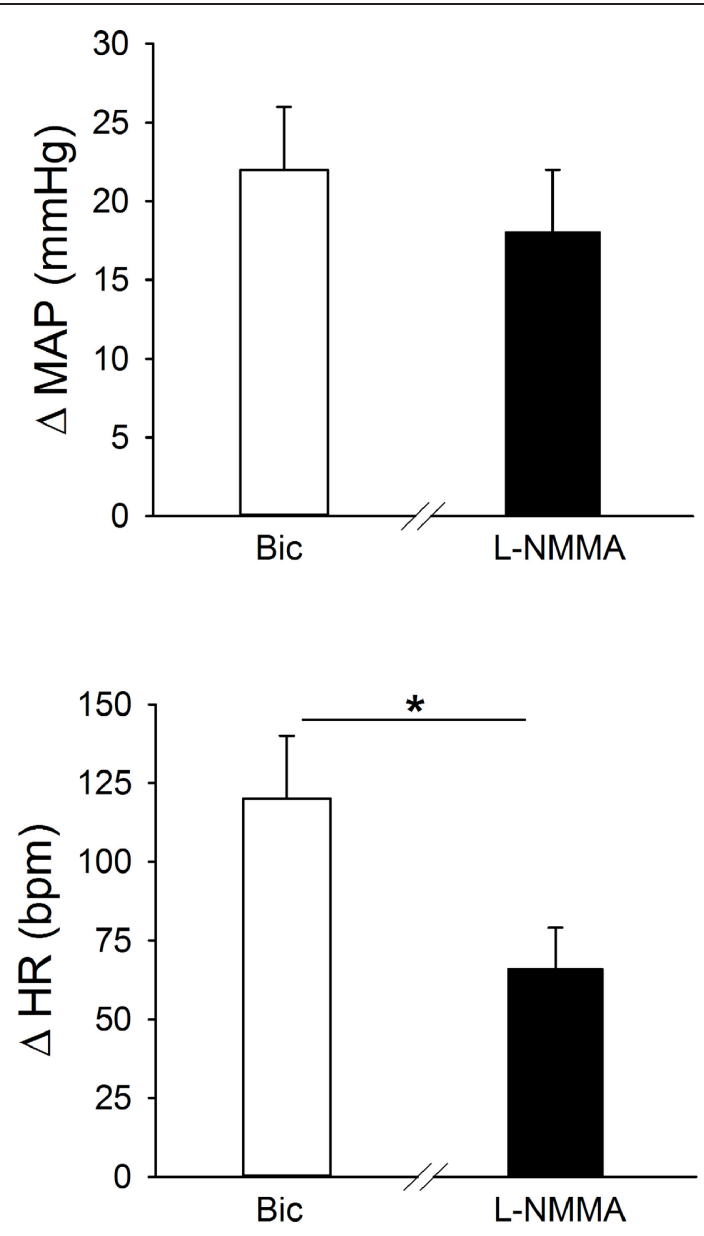

FIGURE 3 | Effects of bilateral blockade in the region of the PVN of $\mathrm{GABA}_{\mathrm{A}}$ receptors (bicuculline; Bic, $1 \mathrm{mM}$; open bars; $n=7$ ), or NO synthase (NOS) (L-NMMA; 2 mM, filled bars; $n=6$ ) on MAP and HR in separate groups of conscious rats. Bilateral microinjection of both Bic and L-NMMA significantly increased MAP and HR. The pressor response to Bic and L-NMMA was similar, while the tachycardic response to L-NMMA was significantly less than the response to Bic $(* p \leq 0.05)$.

of GABA and NO appear to be synergistic. Finally, these studies demonstrate for the first time that under basal conditions in the conscious rat, endogenous $\mathrm{NO}$ attenuates the tonic pressor effects of glutamate within the region of the PVN.

In the PVN a number of excitatory and inhibitory neurotransmitters converge to influence neuronal activity (Swanson and Sawchenko, 1983). Studies in vitro and in anesthetized animals suggest an interaction among glutamate, GABA, and NO in regulation of the PVN (Horn et al., 1994; Zhang and Patel, 1998; Stern, 2004). The current experiments indicate that an interaction between glutamate and GABA, as well as an interaction of NO with both glutamate and GABA, contributes to PVN control of arterial pressure in conscious rats. Taken together, the data suggest that in conscious rats the influence of the PVN on arterial pressure and HR reflects a complex balance among a variety of excitatory and inhibitory inputs. A schematic of these potential interactions is shown in Figure 8 and will be described below. 


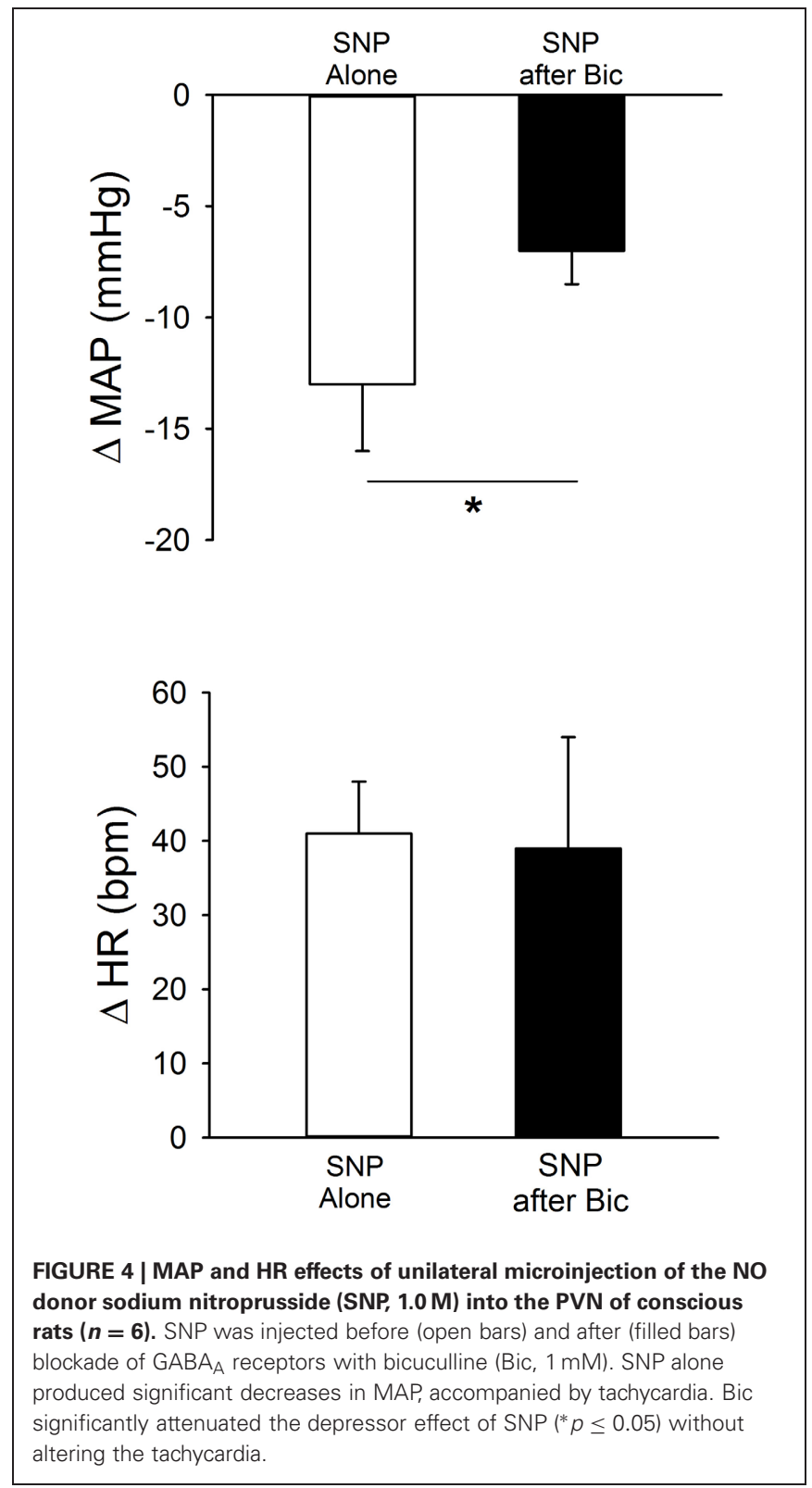

\section{TONIC EFFECTS OF THE PVN}

The PVN is a critical site of neuroendocrine integration and plays an important role in modulating sympathetic tone and arterial pressure under a variety of physiological and pathophysiological conditions including heart failure, diabetes, and hypertension (Patel and Zhang, 1996; Martin and Haywood, 1998; Patel, 2000; DiCarlo et al., 2002; Felder et al., 2003; Li and Patel, 2003; Mueller et al., 2006; Heesch et al., 2009). However, its role in basal cardiovascular control is not entirely clear, although it likely reflects the integration of both excitatory and inhibitory influences. Bilateral inhibition of $\mathrm{GABA}_{\mathrm{A}}$ receptors with Bic in the PVN region of conscious (Martin et al., 1991) or anesthetized (Zhang and Patel, 1998; Chen and Toney, 2003; Chen et al., 2003) rats increases MAP and HR, suggesting that the PVN is under tonic GABAergic inhibition (Figure 8A). The current study provides additional

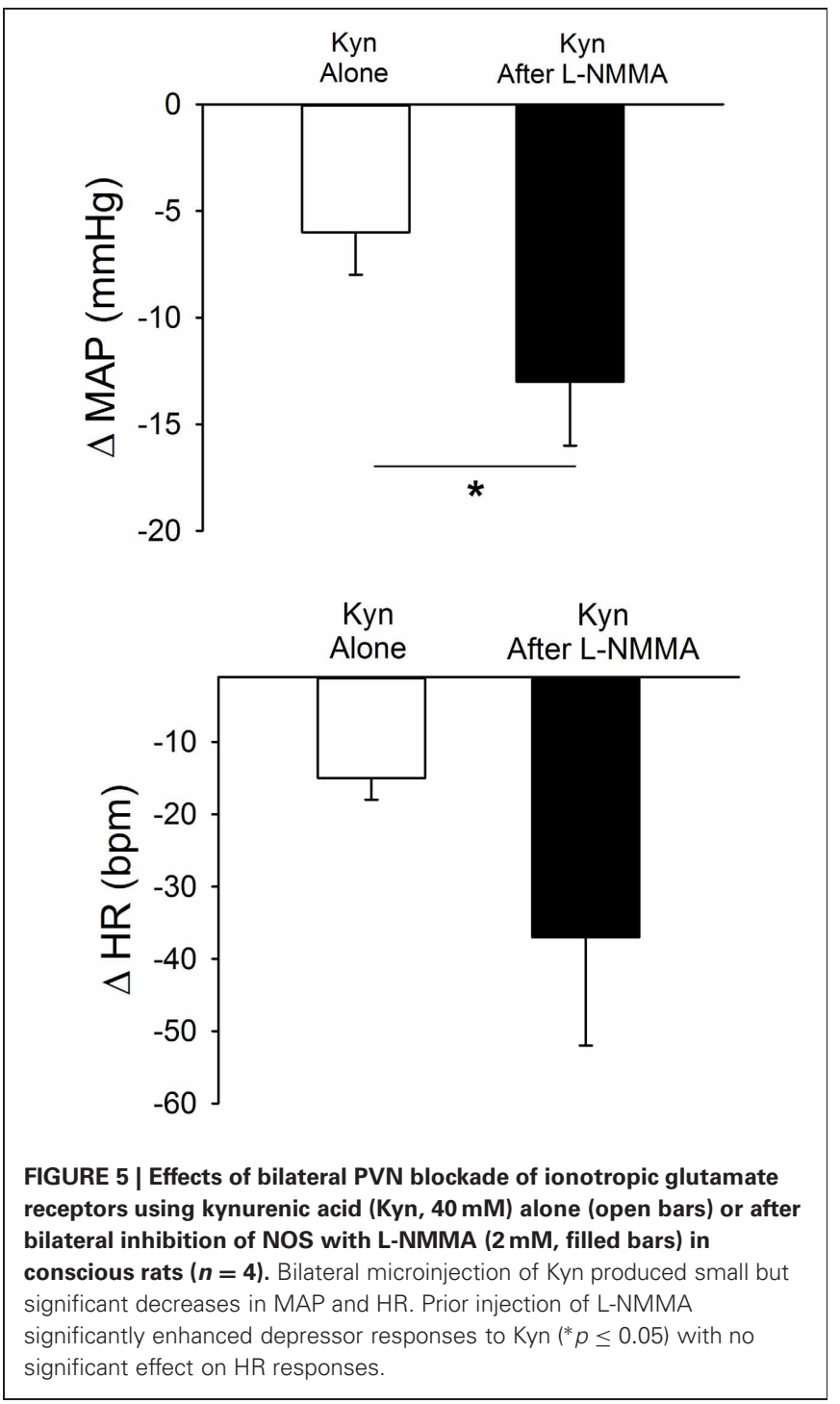

support for tonic GABAergic inhibition in conscious rats and also indicates that the region of the PVN is under tonic inhibition due to NO in the conscious state (Figure 8B). Similar to experiments in anesthetized animals (Zhang and Patel, 1998), bilateral microinjection of the NOS blocker L-NMMA increased MAP and HR. In the present study, we also investigated the contribution of EAAs in the region of the PVN to maintenance of basal arterial pressure and in the response to inhibition of $\mathrm{GABA}_{\mathrm{A}}$ receptors in conscious rats. In our experiments, bilateral blockade of ionotropic glutamate receptors produced a small but significant reduction in arterial pressure and HR, suggesting that the balance of inputs is such that EAAs provide a small tonic excitatory effect within the PVN, which contributes to maintenance of basal arterial pressure and HR (Figure 8C). Similar experiments in anesthetized rats (Chen et al., 2003) and conscious rabbits (Badoer et al., 2002) showed that blockade of ionotropic EAA receptors in the PVN had no significant effect on baseline arterial pressure. The difference in these studies may be due to the presence of anesthesia, unilateral versus bilateral injections, and 


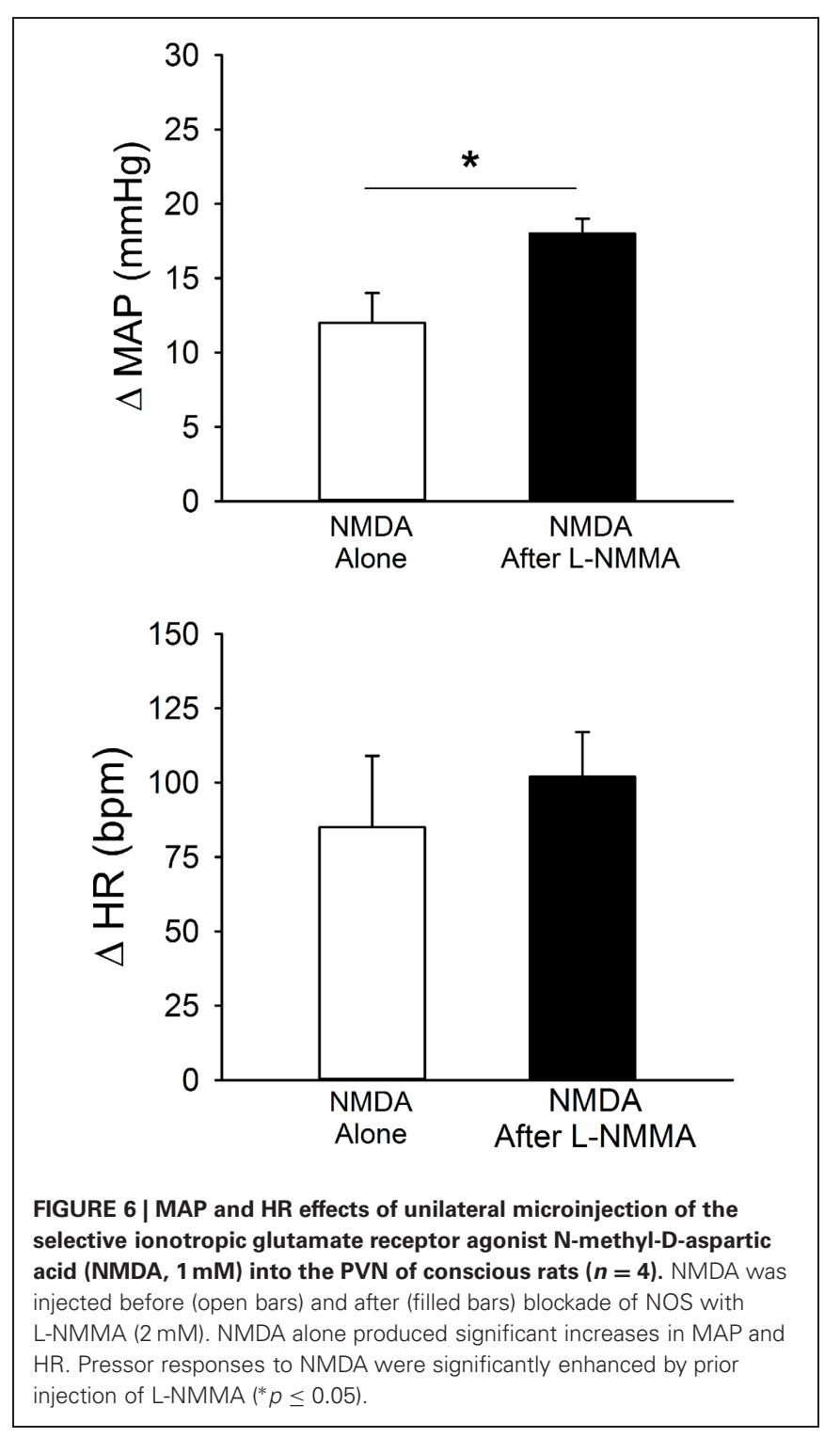

possible species differences. Alternatively, as previously suggested (Badoer et al., 2002), the PVN may exert both excitatory and inhibitory effects on sympathetic nerve activity, and the relative influence of these effects may vary depending on species and state of the animal. Nevertheless, the current data are consistent with the hypothesis that the relatively small tonic excitatory effects exerted by the PVN involve, at least in part, ionotropic glutamatergic transmission. Other potential sources of excitation in the PVN (Figure 8D) were not evaluated in the current experiments. However, previous studies in anesthetized rats suggest that angiotensin II also contributes to excitation of the PVN under both physiological and pathophysiological conditions (Chen and Toney, 2003; Freeman and Brooks, 2007).

\section{NITRIC OXIDE AND GABA}

Unilateral microinjection of the NO donor SNP into the region of the PVN of conscious rats decreased MAP, and blockade of NOS with L-NMMA-induced pressor and tachycardic responses. These
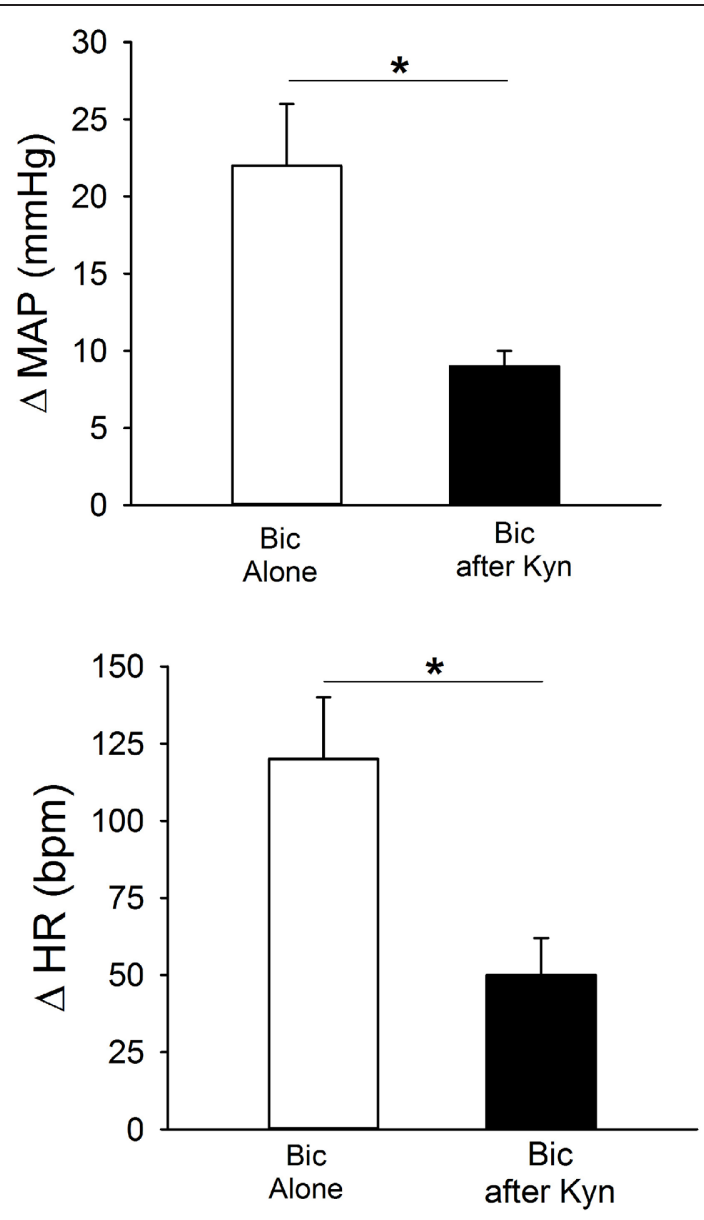

FIGURE 7 | Effects of bilateral blockade within the PVN of GABA receptors with bicuculline (Bic, $1 \mathrm{mM}$ ) on MAP and $\mathrm{HR}$ in conscious rats $(n=6)$ alone (open bars) or after blockade of ionotropic glutamate receptors with kynurenic acid (Kyn, $\mathbf{4 0} \mathrm{mM}$; filled bars). Bilateral microinjection of Bic alone produced significant increases in MAP and HR. Pressor and tachycardic responses to Bic were significantly attenuated by prior microinjection of Kyn $(* p \leq 0.05)$.

sympathoinhibitory effects of NO in the conscious state are consistent with previous work examining responses to SNP (Zhang and Patel, 1998) or NOS blockade (Mastelari et al., 2011) in the PVN. Other studies in conscious Wistar rats reported no effect (Hashiguchi et al., 1997) or pressor responses (Busnardo et al., 2010) to PVN microinjection of NO donors. While the reasons for this discrepancy are not clear, differences including drug concentration and strain could contribute. Taken together, the preponderance of evidence suggests that NO is an inhibitory modulator of cardiovascular control in the PVN in both conscious and anesthetized animals. Microinjection of SNP into the region of the PVN was associated with tachycardia in the present experiments rather than bradycardia as previously reported (Zhang and Patel, 1998; Wang et al., 2005). The mechanism for this tachycardia is not completely clear. It is possible that it was a baroreflex-mediated response to the decrease in arterial pressure. Alternatively, it may be a direct effect of SNP in the PVN rather 


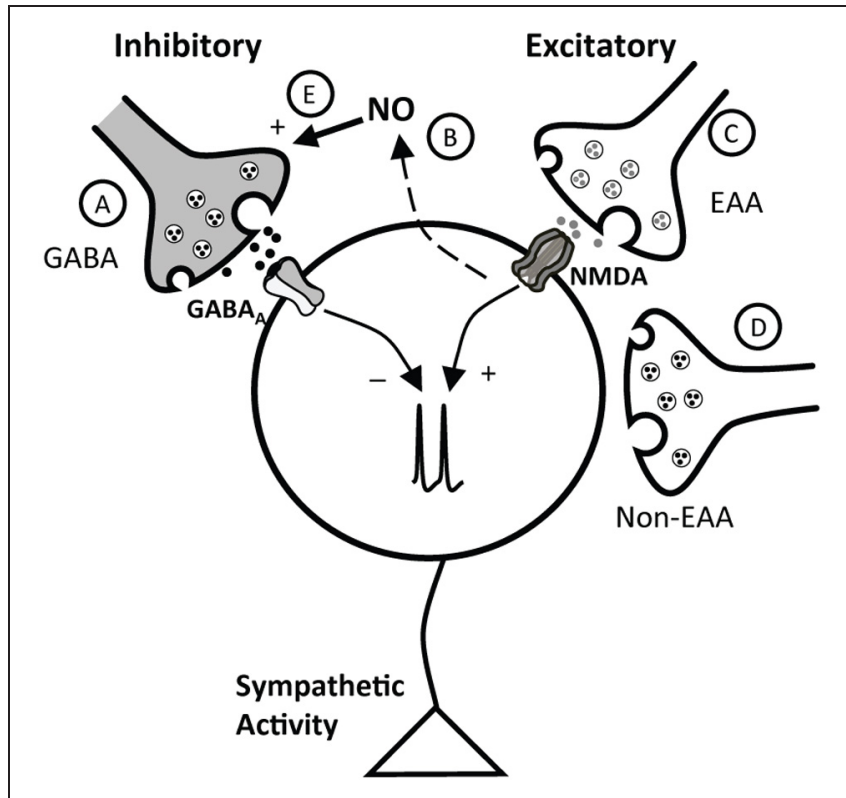

FIGURE 8 | Schematic representation of potential interactions of inhibitory and excitatory inputs to the PVN. The cell in the schematic represents a PVN neuron involved in control of sympathetic nervous system activity. As described in the text, this neuron receives inhibitory influences due to $G A B A$, via $G_{A B A}$ receptors $(\mathbf{A})$, and $N O(B)$, which appears to exert its effects at least in part through GABA (E). Excitatory inputs include both excitatory amino acid (EAA, C) and non-ionotropic EAA receptor mediated (non-EAA) (D) inputs. NO, likely acting through GABA, both blunts the response to activation of NMDA receptors, and tonically inhibits the effects of ongoing EAA excitation.

than baroreflex-mediated, because although the depressor effect of SNP was diminished in the presence of Bic, the tachycardia was unchanged. Thus, the tachycardia likely was not dependent on the magnitude of the depressor response. Furthermore, it does not appear to require $\mathrm{GABA}_{\mathrm{A}}$ receptors, because it was not altered by pretreatment with Bic.

Full expression of the depressor effect of $\mathrm{NO}$ in the PVN region of awake rats appears to require GABAergic transmission (Figure 8E), since responses to SNP were attenuated $\sim 50 \%$ by Bic. In previous work in anesthetized animals (Zhang and Patel, 1998), Bic abolished responses to SNP. Thus, it appears that in the absence of anesthesia, a portion of the response to NO donors is independent of GABA. Another possibility may be related to the dose of Bic used. In the present study, we used a relatively low dose of Bic $(1 \mathrm{mM})$. As reported by others (Martin et al., 1991), we observed that Bic injection into the PVN of conscious rats resulted in mild behavioral responses (such as licking and grooming). We found that higher doses tended to elicit locomotor activity that interfered with measurements of arterial pressure and HR. Therefore, we chose a dose within the lower range of those previously used in an effort to circumvent this concern. It is possible that this relatively low dose of Bic did not completely block $\mathrm{GABA}_{\mathrm{A}}$ receptors. Although previous studies in anesthetized rats indicate that cardiovascular responses to $40 \mathrm{mM}$ and $1 \mathrm{mM}$ Bic microinjected into the PVN were similar (Zhang and Patel, 1998; Li et al., 2006), we cannot completely eliminate the possibility that our $\mathrm{GABA}_{\mathrm{A}}$ receptor blockade was not complete in the current study. Nonetheless, it appears that in conscious rats, NO exerts primarily an inhibitory role in the PVN and this inhibition is dependent at least in part on GABA. However, it is possible that the contribution of GABA to NO-mediated effects may be less in conscious compared to anesthetized animals.

\section{NITRIC OXIDE AND GLUTAMATE}

Excitatory responses to activation of the NMDA subtype of glutamate receptors in the region of the PVN were significantly enhanced by prior blockade of NOS. These data in conscious rats extend previous findings in anesthetized rats (Li et al., 2001) demonstrating that the excitatory effects of exogenously administered NMDA in the PVN are blunted by NO (Figure 8, dashed line). Although not specifically examined in our study, this effect of NO may also involve an effect mediated by GABA (Figure 8E) rather than modulation of glutamatergic signaling, as NO appears to increase directly glutamate release (Horn et al., 1994) and AMPA-mediated currents (Roychowdhury et al., 2006) in the PVN. The effects of NO to modulate GABA function appear to predominate, possibly due to greater tonic GABAergic input on PVN neurons.

We also examined the influence of NO on the effects of tonic endogenous ionotropic glutamate receptor mechanisms within the PVN. The NOS inhibitor L-NMMA enhanced the depressor response to bilateral inhibition of ionotropic glutamate receptors with Kyn. Importantly, the depressor response to Kyn was enhanced even though L-NMMA would be expected to increase arterial pressure over the same time period. These data suggest that in conscious rats not only does NO blunt the response to exogenously administered NMDA, but the endogenous, tonic excitatory effects of glutamate in the PVN also are under tonic negative modulation by endogenous NO. To our knowledge, this is the first demonstration of an effect of endogenous $\mathrm{NO}$ on tonic EAA-mediated excitation within the PVN. Since we also found that the pressor response to exogenous NMDA is potentiated following blockade of NOS, tonic effects of endogenous NO also may involve the NMDA subtype of glutamate receptors. Thus, the overall effect of NO within the PVN is inhibitory, since $\mathrm{NO}$ attenuates tonic excitation due to both exogenous and tonic endogenous glutamate, and facilitates inhibitory GABA neurotransmission.

\section{GABA AND GLUTAMATE}

Endogenous GABA and glutamate also appear to interact in the PVN of conscious rats. Bilateral removal of $\mathrm{GABA}_{\mathrm{A}}$ receptormediated inhibition in the region of the PVN produced pressor and tachycardic responses, effects observed in other studies using conscious rats (Martin et al., 1991; de Abreu et al., 2009). Experiments in anesthetized animals suggest that this response is due to an unmasking of excitatory inputs, including glutamate and angiotensin II (Chen and Toney, 2003; Chen et al., 2003; Li and Pan, 2007a). In the current study, excitatory responses to $\mathrm{GABA}_{\mathrm{A}}$ blockade were blunted during inhibition of ionotropic glutamate receptors. These data suggest that in the conscious rat, glutamate provides a substantial portion of the excitation that is evident when $\mathrm{GABA}_{\mathrm{A}}$ inhibition is removed (Figure 8C), or 
glutamatergic inputs may be tonically inhibited by GABA. In this regard, following Bic in the PVN, release of glutamate and the frequency of glutamatergic EPSCs are increased (Li et al., 2006). Alternatively, GABAergic neurons in the region of the PVN may be excited tonically by glutamate. Although simultaneous disfacilitation due to Kyn could contribute to the blunted response to $\mathrm{Bic}$, this most likely involves an interaction between glutamate and GABA as previously suggested (Chen et al., 2003) because the minor effects of Kyn alone are not sufficient to account for the magnitude of the diminished response. Future experiments are required to evaluate the cellular mechanisms of these interactions, as well as a potential role for other transmitters such as angiotensin II.

\section{LIMITATIONS}

As with any study our conclusions have certain caveats that must be taken into consideration. For example, in the current study we measured arterial pressure and HR in conscious rats. It is most likely that responses to microinjections are due primarily to changes in sympathetic nervous system activity. Although this assumption is consistent with previous studies in anesthetized animals (Zhang and Patel, 1998; Li et al., 2001), numerous neural and humoral factors influence arterial pressure, and the influence of the PVN on sympathetic activity may vary depending on the specific sympathetic nerve evaluated. Therefore, future experiments are necessary to confirm the involvement of the sympathetic nervous system, including effects on sympathetic activity to specific regions, vs. other neurohumoral factors.

Also, because of non-specific damage during removal of guide cannulae, we cannot say unequivocally that all injections were centered in the PVN. However, injection sites were confined to the brain parenchyma, and the primary cardiovascular region nearest the injections was in fact the PVN. In all rats, consistent with

\section{REFERENCES}

Akine, A., Montanaro, M., and Allen, A. M. (2003). Hypothalamic paraventricular nucleus inhibition decreases renal sympathetic nerve activity in hypertensive and normotensive rats. Auton. Neurosci. 108, 17-21.

Araújo, G. C., Lopes, O. U., and Campos, R. R. Jr. (1999). Importance of glycinergic and glutamatergic synapses within the rostral ventrolateral medulla for blood pressure regulation in conscious rats. Hypertension 34, 752-755.

Badoer, E. (1996). Cardiovascular role of parvocellular neurons in the paraventricular nucleus of the hypothalamus. Physiology 11, 43-47.

Badoer, E., Ng, C. W., and De, M. R. (2002). Tonic sympathoinhibition arising from the hypothalamic PVN in the conscious rabbit. Brain Res. 947, 17-24.

Bredt, D. S., Hwang, P. M., and Snyder, S. H. (1990). Localization of nitric oxide synthase indicating a neural role for nitric oxide. Nature 347, 768-770.

Busnardo, C., Crestani, C. C., Tavares, R. F., Resstel, L. B., and Correa, F. M. (2010). Cardiovascular responses to L-glutamate microinjection into the hypothalamic paraventricular nucleus are mediated by a local nitric oxide-guanylate cyclase mechanism. Brain Res. 1344, 87-95.

Chen, Q. H., Haywood, J. R., and Toney, G. M. (2003). Sympathoexcitation by PVNinjected bicuculline requires activation of excitatory amino acid receptors. Hypertension 42, 725-731.

Chen, Q. H., and Toney, G. M. (2001). $\mathrm{AT}_{1}$-receptor blockade in the hypothalamic PVN reduces central hyperosmolality-induced renal sympathoexcitation. Am. J. Physiol. Regul. Integr. Comp. Physiol. 281, R1844-R1853.

responses in the PVN, disinhibition (Bic) or excitation (NMDA) produced pressor responses with limited behavioral activation. In addition, our coordinates are consistent with those used previously by us, in which injection sites were confirmed (Kvochina et al., 2009; King et al., 2012). Collectively, this evidence suggests that responses were due primarily to changes in neuronal activation in the region of the PVN.

\section{SUMMARY}

The combined data from these studies in conscious rats is consistent with previous work in anesthetized animals and suggests that the PVN is under tonic excitation due to endogenous activation of ionotropic glutamate receptors. In addition, both NO and GABA have tonic inhibitory effects in the region of the PVN in conscious rats and this inhibition masks the majority of tonic glutamatergic excitation. Importantly, we found that in conscious rats, endogenous NO appears to tonically blunt the ongoing activation of the PVN by EAAs. The nature of these tonic effects involves a complex interaction among glutamatergic, GABAergic, and NO mechanisms.

\section{ACKNOWLEDGMENTS}

The authors would like to acknowledge the outstanding technical expertise of Sarah A. Friskey. We would also like to thank the Neurohumoral Control of the Circulation group at the Dalton Cardiovascular Research Center for valuable input on this project. This study was supported by the National Institutes of Health HL 55306 and HL 64669 (Eileen M. Hasser). This investigation was conducted in a facility constructed with support from Research Facilities Improvement Program Grant Number CO6 RR-16498 from the National Center for Research Resources, National Institutes of Health.

Chen, Q. H., and Toney, G. M. (2003). Responses to GABA-A receptor blockade in the hypothalamic PVN are attenuated by local $\mathrm{AT}_{1}$ receptor antagonism. Am. J. Physiol. Regul. Integr. Comp. Physiol. 285, R1231-R1239.

Coote, J. H. (2004). A role for the paraventricular nucleus of the hypothalamus in the autonomic control of heart and kidney. Exp. Physiol. 90, 169-173.

de Abreu, S. B., Lenhard, A., Mehanna, A., de Souza, H. C. D., de Aguiar Correa, F. M., Hasser, E. M., et al. (2009). Role of paraventricular nucleus in exercise traininginduced autonomic modulation in conscious rats. Auton. Neurosci. 148, 28-35.

DiCarlo, S. E., Zheng, H., Collins, H. L., Rodenbaugh, D. W., and Patel, K. P. (2002). Daily exercise normalizes the number of diaphorase (NOS) positive neurons in the hypothalamus of hypertensive rats. Brain Res. 955, 153-160.

Felder, R. B., Francis, J., Zhang, Z. H., Wei, S. G., Weiss, R. M., and Johnson, A. K. (2003). Heart failure and the brain: new perspectives. Am. J. Physiol. Regul. Integr. Comp. Physiol. 284, R259-R276.

Franks, N. P., and Lieb, W. R. (1982). Molecular mechanisms of general anaesthesia. Nature 300, 487-493.

Freeman, K. L., and Brooks, V. L. (2007). AT1 and glutamatergic receptors in paraventricular nucleus support blood pressure during water deprivation. Am. J. Physiol. Regul. Integr. Comp. Physiol. 292, R1675-R1682.

Hardy, S. G. P. (2001). Hypothalamic projections to cardiovascular centers of the medulla. Brain Res. 894, 233-240.

Hashiguchi, H., Ye, S. H., RossCisneros, F., and Alexander, N. (1997). Central nitric oxide donors attenuate cardiovascular and central 
norepinephrine responses to stress. Am. J. Physiol. Regul. Integr. Comp. Physiol. 272, R1447-R1453.

Heesch, C. M., Zheng, H., Foley, C. M., Mueller, P. J., Hasser, E. M., and Patel, K. P. (2009). Nitric oxide synthase activity and expression are decreased in the paraventricular nucleus of pregnant rats. Brain Res. 1251, 140-150.

Hirooka, Y., Polson, J. W., and Dampney, R. A. L. (1996). Pressor and sympathoexcitatory effects of nitric oxide in the rostral ventrolateral medulla. J. Hypertens. 14, 1317-1324.

Horn, T., Smith, P. M., McLaughlin, B. E., Bauce, L., Marks, G. S., Pittman, Q. J., et al. (1994). Nitric oxide actions in paraventricular nucleus: cardiovascular and neurochemical implications. Am. J. Physiol. Regul. Integr. Comp. Physiol. 266, R306-R313.

Jin, Y. H., Zhang, Z., Mendelowitz, D., and Andresen, M. C. (2009). Presynaptic actions of propofol enhance inhibitory synaptic transmission in isolated solitary tract nucleus neurons. Brain Res. 1286, 75-83.

Kannan, H., Hayashida, Y., and Yamashita, H. (1989). Increase in sympathetic outflow by paraventricular nucleus stimulation in awake rats. Am. J. Physiol. 256, R1325-R1330.

Kannan, H., Niijima, A., and Yamashita, H. (1987). Inhibition of renal sympathetic nerve activity by electrical stimulation of the hypothalamic paraventricular nucleus in anesthetized rats. J. Auton. Nerv. Syst. 21, 83-86.

Kantzides, A., and Badoer, E. (2005) nNos-containing neurons in the hypothalamus and medulla project to the RVLM. Brain Res. 1037, 25-34.

Kenney, M. J., Weiss, M. L., Patel, K. P., Wang, Y., and Fels, R. J. (2001). Paraventricular nucleus bicuculline alters frequency components of symapthetic nerve discharge bursts. Am. J. Physiol. Heart Circ. Physiol. 281, H1233-H1241.

King, T. L., Heesch, C. M., Clark, C. G., Kline, D. D., and Hasser, E. M. (2012). Hypoxia activates nucleus tractus solitarii neurons projecting to the paraventricular nucleus of the hypothalamus. Am. J. Physiol. Regul. Integr. Comp. Physiol. 302, R1219-R1232.

Kvochina, L., Hasser, E. M., and Heesch, C. M. (2009). Pregnancy decreases GABAergic inhibition of the hypothalamic paraventricular nucleus. Physiol. Behav. 97, 171-179.

Li, D. P., and Pan, H. L. (2007a). Glutamatergic inputs in the hypothalamic paraventricular nucleus maintain sympathetic vasomotor tone in hypertension. Hypertension 49, 916-925.

Li, D. P., and Pan, H. L. (2007b). Role of $\gamma$-aminobutyric acid (GABA)A and GABAB receptors in paraventricular nucleus in control of sympathetic vasomotor tone in hypertension. J. Pharmacol. Exp. Ther. 320, 615-626.

Li, Y.-F., Jackson, K. L., Stern, J. E., Rabeler, B., and Patel, K. P. (2006). Interaction between glutamate and GABA systems in the integration of sympathetic outflow by the paraventricular nucleus of the hypothalamus. Am. J. Physiol. Heart Circ. Physiol. 291, 2847-2856.

Li, Y.-F., Mayhan, W. G., and Patel, K. P. (2001). NMDA-mediated increase in renal sympathetic nerve discharge within the PVN: role of nitric oxide. Am. J. Physiol. Heart Circ. Physiol. 281, H2328-H2336.

Li, Y.-F., and Patel, K. P. (2003). Paraventricular nucleus of the hypothalamus and elevated sympathetic activity in heart failure: the altered inhibitory mechanisms. Acta Physiol. Scand. 177, 17-26.

Li, Y., Zhang, W., and Stern, J. E. (2003). Nitric oxide inhibits the firing activity of hypothalamic paraventricular neurons that innervate the medulla oblongata: role of GABA. Neuroscience 118, 585-601.

Machado, B. H. (2001). Neurotransmission of the cardiovascular reflexes in the nucleus tractus solitarii of awake rats. Ann. N.Y. Acad. Sci. 940, 179-196.

Martin, D. S., and Haywood, J. R. (1998). Reduced GABA inhibition of sympathetic function in renalwrapped hypertensive rats. Am. J. Physiol. Regul. Integr. Comp. Physiol. 275, R1523-R1529.

Martin, D. S., Segura, T., and Haywood, J. R. (1991). Cardiovascular responses to bicuculline in the paraventricular nucleus of the rat. Hypertension 18, 48-55.

Martins-Pinge, M. C., Baraldi-Passy, I., and Lopes, O. U. (1997). Excitatory effects of nitric oxide within the rostral ventorlateral medulla of freely moving rats. Hypertension 30 , 704-707.

Mastelari, R. B., de Souza, H. C., Lenhard, A., de Aguiar Correa, F. M., and Martins-Pinge, M. C. (2011). Nitric oxide inhibition in paraventricular nucleus on cardiovascular and autonomic modulation after exercise training in unanesthetized rats. Brain Res. 1375, 68-76.

Moffitt, J. A., Foley, C. M., Schadt, J. C., Laughlin, M. H., and Hasser, E. M. (1998). Attenuated baroreflex control of sympathetic nerve activity after cardiovascular deconditioning in rats. Am. J. Physiol. Regul. Integr. Comp. Physiol. 274, R1397-R1405.

Moffitt, J. A., Schadt, J. C., and Hasser, E. M. (1999). Altered central nervous system processing of baroreceptor input following hindlimb unloading in rats. Am. J. Physiol. Heart Circ. Physiol. 277, H2272-H2279.

Mueller, P. J., Cunningham, J. T., Patel, K. P., and Hasser, E. M. (2003). Proposed role of the paraventricular nucleus in cardiovascular deconditioning. Acta Physiol. Scand. 177, 27-35.

Mueller, P. J., Foley, C. M., Heesch, C. M., Cunningham, J. T., Zheng, H., Patel, K. P., et al. (2006). Increased nitric oxide synthase activity and expression in the hypothalamus of hindlimb unloaded rats. Brain Res. 1115, 65-74.

Olsen, R., and Li, G. D. (2011). GABA(A) receptors as molecular targets of general anesthetics: identification of binding sites provides clues to allosteric modulation. Can. J. Anaesth. 58, 206-215.

Patel, K. P. (2000). Role of paraventricular nucleus in mediating sympathetic outflow in heart failure. Heart Fail. Rev. 5, 73-86.

Patel, K. P., Li, Y.-F., and Hirooka, Y. (2001). Role of nitric oxide in central sympathetic outflow. Exp. Biol. Med. 226, 814-824.

Patel, K. P., and Zhang, K. (1996). Neurohumoral activation in heart failure: role of paraventricular nucleus. Clin. Exp. Pharmacol. Physiol. 23, 722-726.

Paxinos, G., and Watson, C. (2007). The Rat Brain in Stereotaxic Coordinates. San Diego, CA: Academic Press.

Pyner, S., and Coote, J. H. (2000). Identification of branching paraventricular neurons of the hypothalamus that project to the rostroventrolateral medulla and spinal cord. Neuroscience 100, 549-556.

Roychowdhury, S., Noack, J., Engelmann, M., Wolf, G., and Horn, T. F. (2006). AMPA receptorinduced intracellular calcium response in the paraventricular nucleus is modulated by nitric oxide: calcium imaging in a hypothalamic organotypic cell culture model. Nitric Oxide 14, 290-299.
Sakima, A., Yamazato, M., Sesoko, S., Muratani, H., and Fukiyama, K. (2000). Cardiovascular and sympathetic effects of L-Glutamate and glycine injected into the rostral ventrolateral medulla of conscious rats. Hypertens. Res. 23, 633-641.

Saper, C. B., Loewy, A. D., Swanson, L. W., and Cowan, W. M. (1976). Direct hypothalamo-autonomic connections. Brain Res. 117, 305-312.

Schadt, J. C., and Ludbrook, J. (1991). Hemodynamic and neurohumoral responses to acute hypovolemia in conscious mammals. Am. J. Physiol. 260, H305-H318.

Shekhar, A., and DiMicco, J. A. (1987). Defense reaction elicited by injection of GABA antagonists and synthesis inhibitors into the posterior hypothalamus in rats. Neuropharmacology 26, 407-417.

Stern, J. E. (2004). Nitric oxide and homeostatic control: an intercellular signalling molecule contributing to autonomic and neuroendocrine integration. Prog. Biophys. Mol. Biol. 84, 197-215.

Swanson, L. W., and Sawchenko, P. E. (1983). Hypothalamic integration: organization of the paraventricular and supraoptic nuclei. Annu. Rev. Neurosci. 6, 269-324.

Villar, M. J., Ceccatelli, S., Ronnqvist, M., and Hokfelt, T. (1994). Nitric oxide synthase increases in hypothalamic magnocellular neurons after salt loading in the rat. An immunohistochemical and in situ hybridization study. Brain Res. 644, 273-281.

Wang, Y., Lui, X. F., Cornish, K. G., Zucker, I. H., and Patel, K. P. (2005). Effects of nNOS antisense in the paraventricular nucleus on blood pressure and heart rate in rats with heart failure. Am. J. Physiol. Heart Circ. Physiol. 288, H205-H213.

Wible, J. H. Jr., Luft, F. C., and DiMicco, J. A. (1988). Hypothalamic GABA suppresses sympathetic outflow to the cardiovascular system. Am. J. Physiol. Regul. Integr. Comp. Physiol. 254, R680-R687.

Zanzinger, J. (1999). Role of nitric oxide in the neural control of cardiovascular function. Cardiovasc. Res. 43, 639-649.

Zhang, K., and Patel, K. P. (1998). Effect of nitric oxide within the paraventricular nucleus on renal sympathetic nerve discharge: role of GABA. Am. J. Physiol. Regul. Integr. Comp. Physiol. 275, R728-R734.

Zhang, K., Zucker, I. H., and Patel, K. P. (1998). Altered number of 
diaphorase (NOS) positive neurons in the hypothalamus of rats with heart failure. Brain Res. 786, 219-225.

Zhang, Z.-H., Francis, J., Weiss, R. M., and Felder, R. B. (2002). The reninangiotensin-aldosterone system excites hypothalamic paraventricular nucleus neurons in heart failure. Am. J. Physiol. Heart Circ. Physiol. 283, H423-H433.
Conflict of Interest Statement: The authors declare that the research was conducted in the absence of any commercial or financial relationships that could be construed as a potential conflict of interest.

Received: 28 July 2012; accepted: 17 December 2012; published online: 09 January 2013.
Citation: Martins-Pinge MC, Mueller PJ, Foley CM, Heesch CM and Hasser EM (2013) Regulation of arterial pressure by the paraventricular nucleus in conscious rats: interactions among glutamate, GABA, and nitric oxide. Front. Physio. 3:490. doi: 10.3389/fphys. 2012.00490

This article was submitted to Frontiers in Integrative Physiology, a specialty of Frontiers in Physiology.
Copyright (C) 2013 Martins-Pinge Mueller, Foley, Heesch and Hasser. This is an open-access article distributed under the terms of the Creative Commons Attribution License, which permits use, distribution and reproduction in other forums, provided the original authors and source are credited and subject to any copyright notices concerning any third-party graphics etc. 